Psychother Psychosom 2017;86:54-56

DOI: $10.1159 / 000447541$

\section{Effects of the Integrative Mind-Body Intervention on Depression, Sleep Disturbances and Plasma IL-6}

Celia H.Y. Chan a, d, Xiao-Wen Jia, Jessie S.M. Chan a, Bobo H.P. Lau, Kwok-Fai So ${ }^{\mathrm{b}}$, Ang Lib , Ka-Fai Chung c, d, Siu-man Nga, Cecilia L.W. Chan ${ }^{\mathrm{a}, \mathrm{d}}$

Departments of a Social Work and Social Administration, b Ophthalmology and ${ }^{\mathrm{C}}$ Psychiatry, and ${ }^{\mathrm{d}}$ Centre on Behavioral Health, The University of Hong Kong, Hong Kong, China

Poor sleep quality is a diagnostic criterion in major depressive disorder [1]. Individuals with depression tend to suffer from sleep disturbances, including difficulty in initiating or maintaining asleep, early morning awakening, unrefreshing sleep, and hypersomnia. A growing body of research also suggests that inflammatory cytokines, such as interleukin-6 (IL-6), are associated with the manifestation of depression and sleep disturbances [2, 3]. Although empirical findings regarding the direction of association among depression, sleep disturbances, and IL- 6 are not conclusive $[2,3]$, their association underscores the interconnectivity between the body and the mind.
The National Center for Complementary and Integrative Health (NCCIH) defines mind-body medicine as a variety of techniques designed 'to use the mind to affect physical functioning and promote health' [4]. The integrative body-mind-spirit (I-BMS) model is an integrative approach derived from Traditional Chinese Medicine, Confucianism, and Daoism [5]. Core beliefs of the IBMS model include (a) the interconnectedness of body, mind, and spirit, (b) the importance of spirituality as a domain of human existence, (c) the need to reach beyond symptom reduction to attain growth and transformative changes and (d) the dual goal of healing and capacity building [6]. Numerous studies have supported its effectiveness in enhancing the quality of life of various clinical populations [5-7]. This study attempted to establish the clinical effectiveness of the I-BMS model on sleep disturbances, mood, and inflammatory responses. We hypothesize that compared to the waitlist control (WLC) condition participants in the immediate I-BMS condition will experience greater improvement in depression and sleep disturbances and a reduction in IL-6 levels.

The current study was a randomized, WLC trial (http://www. hkclinicaltrials.com/; trial No. HKCTR-1929; ethical ref. No. UW 13-485). A total of 1,002 Hong Kong Chinese adults from the community completed online a battery of self-administered scales, including the Center for Epidemiologic Studies Depression Scale (CESD), the Pittsburgh Sleep Quality Index (PSQI), and other psychosocial measures. Individuals who were Chinese adults with mild to moderate depression $(34 \geq \operatorname{CESD} \geq 10[8])$ and insomnia

Table 1. Treatment outcomes for sleep, depression, and IL-6 measures

\begin{tabular}{|c|c|c|c|c|c|}
\hline & pretreatment & posttreatment & 3-month follow-up & pre- to posttreatment & $\begin{array}{l}\text { pretreatment to } 3 \text {-month } \\
\text { follow-up }\end{array}$ \\
\hline CESD & & & & $0.37(0.08 \text { to } 0.66)^{\mathrm{a}}$ & $0.44(0.15 \text { to } 0.73)^{\mathrm{a}}$ \\
\hline I-BMS & $21.79 \pm 6.96$ & $15.24 \pm 8.52$ & $13.98 \pm 7.44$ & $0.84(0.42 \text { to } 1.27)^{\mathrm{b}}$ & $1.08(0.65 \text { to } 1.52)^{\mathrm{b}}$ \\
\hline Control & $20.68 \pm 6.16$ & $18.21 \pm 7.66$ & $17.22 \pm 7.39$ & $0.36(-0.05 \text { to } 0.77)^{\mathrm{c}}$ & $0.47(0.10 \text { to } 0.92)^{\mathrm{c}}$ \\
\hline I-BMS & $13.54 \pm 2.80$ & $11.08 \pm 3.79$ & $10.55 \pm 3.78$ & $0.74(0.32 \text { to } 1.16)^{\mathrm{b}}$ & $0.90(0.47 \text { to } 1.33)^{b}$ \\
\hline Control & $13.32 \pm 2.89$ & $12.06 \pm 2.77$ & $11.78 \pm 3.84$ & $0.45(0.03 \text { to } 0.86)^{\mathrm{c}}$ & $0.45(0.04 \text { to } 0.87)^{\mathrm{c}}$ \\
\hline$I L-6$ & & & & $0.46(0.17 \text { to } 0.75)^{\mathrm{a}}$ & $-0.06(-0.34 \text { to } 0.23)^{\mathrm{a}}$ \\
\hline I-BMS & $1.49 \pm 1.95$ & $1.24 \pm 1.70$ & $1.60 \pm 2.93$ & $0.14(-0.27 \text { to } 0.55)^{\mathrm{b}}$ & \\
\hline Control & $1.75 \pm 2.88$ & $2.67 \pm 4.04$ & $1.46 \pm 2.08$ & $-0.26(-0.67 \text { to } 0.15)^{\mathrm{c}}$ & \\
\hline
\end{tabular}

Baseline, posttreatment, and follow-up data are presented for each group along with change scores and within-group effect sizes. Intention-to-treat sample: I-BMS $\mathrm{n}=92, \mathrm{WLC} \mathrm{n}=93$.

${ }^{a}$ Between-group effect size. ${ }^{b}$ Within-group effect size of I-BMS. ${ }^{c}$ Within-group effect size of WLC. For IL-6, a significant betweengroup effect was only attained at posttreatment, but not at the 3-month follow-up. Thus, the within-group effect sizes of IL- 6 are not calculated at the comparison between $\mathrm{T}_{0}$ and $\mathrm{T}_{2}$ in both groups.

\section{KARGER}

(C) 2016 S. Karger AG, Basel

E-Mail karger@karger.com

www.karger.com/pps
Celia H.Y. Chan or Cecilia L.W. Chan

Department of Social Work and Social Administration, University of Hong Kong Pokfulam

Hong Kong (China)

E-Mail chancelia@hku.hk or cecichan@hku.hk 


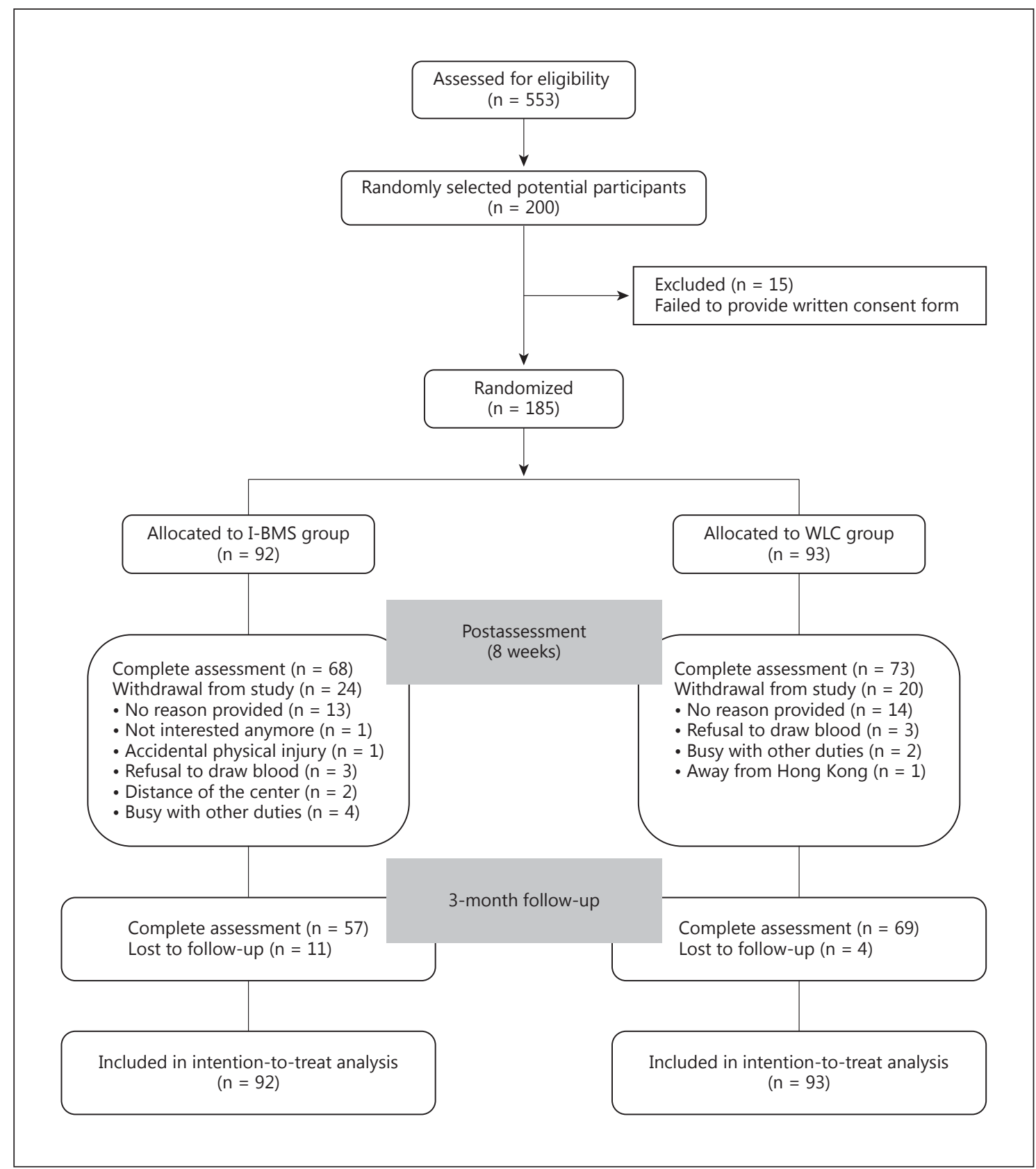

Fig. 1. Flowchart of the randomized controlled trial of I-BMS on depression and sleep disturbances.

(PSQI $>5$ [9]) were recruited. Exclusion criteria included self-reported comorbidity with psychotic conditions such as schizophrenia and previous experience with I-BMS. Of the 533 eligible participants, 200 were randomly selected based on a computergenerated list of random numbers because of resource limitations in intervention. Fifteen participants did not provide written consent, and the remaining 185 participants were randomly assigned to either an immediate I-BMS group $(n=92)$ or a 6 -month WLC condition $(\mathrm{n}=93)$. The I-BMS group consisted of eight 3 -hour weekly group sessions with culturally relevant mind-body exercises, mindfulness practices, self-reflection via experimental activities, and group discussion and sharing. All measures, including the plasma IL-6 levels via blood test, were collected at baseline $\left(\mathrm{T}_{0}\right)$, immediately after the intervention $\left(\mathrm{T}_{1}\right)$, and 3 months after the intervention $\left(\mathrm{T}_{2}\right)$. The flowchart of the trial is presented in figure 1 .

$t$ tests and $\chi^{2}$ tests were used to compare the baseline characteristics of participants in the I-BMS versus WLC groups. Intention- 
to-treat analysis was conducted to evaluate treatment effectiveness. Missing data in the I-BMS group were imputed by last observation score and assuming no improvement. The group mean was used to substitute missing values for participants from the WLC group at $\mathrm{T}_{1}$ and $\mathrm{T}_{2}$. The effects of I-BMS intervention were investigated by ANCOVAs with age, gender, and baseline scores as covariates. Cohen's $d s$ were calculated as effect sizes. Forward linear regression analyses of subsamples were performed to examine the effects of I-BMS on changes of PSQI, CESD (minus the item on sleep quality), and IL- 6 at $\mathrm{T}_{1}$ and $\mathrm{T}_{2}$ for participants with baseline CESD $\geq 16$, indicating significant depressive symptoms [8]. The change of each outcome $\left(\mathrm{T}_{0}-\mathrm{T}_{1}\right)$ was entered as the dependent variable, whereas the treatment conditions (I-BMS vs. WLC) were the predicting variables, with adjustment for age, gender, baseline scores, and the other two key outcomes.

In this study, $75.1 \%$ were female, with a mean age of $55.28 \pm$ 9.83 years. $58.4 \%$ had secondary level education, and $72.4 \%$ were married or cohabited. A majority (93.7\%) of participants reported insomnia that lasted more than 1 year, with $61.1 \%$ reporting more than 3 years. Baseline characteristics, including the scores for key outcomes, were comparable between the two groups (I-BMS vs. WLC) and between dropouts and completers.

Table 1 displays the descriptive statistics and the within- and between-group effect sizes of the two groups at the three time points. The remission rate of depression (a reduction in CES-D score to $<16[8]$ ) was significantly greater in the I-BMS group than in the WLC group at $\mathrm{T}_{1}[45 \%(23 / 51)$ vs. $21 \%(11 / 52)$, difference $=24 \%, 95 \%$ CI: $5.8-40.1 \% ; \mathrm{p}=0.01]$ and $\mathrm{T}_{2}[57 \%(23 / 40)$ vs. $28 \%(13 / 46)$, difference $=29 \%$, 95\% CI: 8-47\%; $\mathrm{p}=0.004]$. ANCOVAs yielded significant group effects on overall sleep quality $\left(\mathrm{F}_{1,180}=2.06, \mathrm{p}=0.016\right)$, depression $\left(\mathrm{F}_{1,180}=13.49, \mathrm{p}<0.001\right)$, and IL-6 $\left(\mathrm{F}_{1,180}=9.02, \mathrm{p}=0.003\right)$ at $\mathrm{T}_{1}$, favoring the intervention group. The effects on overall sleep quality $\left(\mathrm{F}_{1,180}=10.03, \mathrm{p}=0.002\right)$ and depression $\left(\mathrm{F}_{1,180}=13.39, \mathrm{p}<0.001\right)$ but not IL-6 $\left(\mathrm{F}_{1,180}=\right.$ $5.50, \mathrm{p}=0.225)$ were sustained at $\mathrm{T}_{2}$. The effect sizes were small to medium (Cohen's $d$ ranging from 0.30 to 0.46 ). Within-group effect sizes indicate medium to large decreases in PSQI and CESD in the I-BMS group at both $\mathrm{T}_{1}$ and $\mathrm{T}_{2}$. As for IL-6, a medium withingroup effect size was shown at $\mathrm{T}_{1}$ in the I-BMS group.

Results of regression at both $T_{1}(n=103)$ and $T_{2}(n=83)$ show that I-BMS intervention (WLC $=1 ; \mathrm{I}-\mathrm{BMS}=2$ ) was independently associated with an improvement of PSQI at $\mathrm{T}_{2}$ but not at $\mathrm{T}_{1}\left(\mathrm{~T}_{1}\right.$ : $\left.\mathrm{B}=0.98, \mathrm{p}=0.12 ; \mathrm{T}_{2}: \mathrm{B}=1.66, \mathrm{p}=0.02\right)$. The effect of I-BMS intervention on CESD was found to be significant at $T_{1}$ and $T_{2}\left(T_{1}\right.$ : $\left.\mathrm{B}=5.499, \mathrm{p}<0.001 ; \mathrm{T}_{2}: \mathrm{B}=4.05, \mathrm{p}=0.04\right)$, but for IL- 6 the adjusted treatment effects were only found at $\mathrm{T}_{1}\left(\mathrm{~T}_{1}: \mathrm{B}=2.04, \mathrm{p}=\right.$ $0.01 ; \mathrm{T}_{2}: \mathrm{B}=-0.34, \mathrm{p}=0.11$ ).

The current study is the first attempt to test the effect of I-BMS on inflammatory responses alongside other physical (sleep) and mental (depression) health indicators. Our findings indicate the effectiveness of I-BMS in facilitating sleep and alleviating depression, as well as reducing IL- 6 levels (at $\mathrm{T}_{1}$ only). In line with previous findings, the current results support the interconnectivity between the body and the mind [2,3], as well as the efficacy of I-BMS in relieving distress in these two reciprocating faculties of human existence [5-7]. The lack of effect of IL- 6 at $\mathrm{T}_{2}$ could be due to nor- mal fluctuation and variations of plasma IL-6 concentration of the participants.

The current study has several limitations. First, the lack of an active control group compromised the validation of effectiveness. Waitlist control design is limited in detecting specific therapeutic factors that are distinctive from expectation and placebo effects. Future studies may adopt mind-body education or physical exercise programs as active control for examining the therapeutic effect of I-BMS. Second, due to the lack of a standardized diagnostic interview, caution should be taken when generalizing the current findings to a clinical sample. Moreover, insomnia symptoms were collected mainly via subjective reports. Future studies may supplement subjective reports with objective measures, including actigraphy and polysomnography [10]. Currently, there is not sufficient empirical support for the causal direction of the relationships among sleep, mood, and IL-6 [2, 3]. Further intervention and longitudinal studies are needed to provide information on this mindbody relationship by investigating the mediating pathways in the improvement of these indicators.

\section{Disclosure Statement}

This was not an industry-supported study. All authors report no financial conflicts of interest. There was no investigational or off-label use.

\section{References}

1 American Psychiatric Association: Diagnostic and Statistical Manual of Mental Disorders, ed 5. Washington, American Psychiatric Association, 2013.

2 Hannestad J, DellaGioia N, Bloch M: The effect of antidepressant medication treatment on serum levels of inflammatory cytokines: a metaanalysis. Neuropsychopharmacology 2011;36:2452-2459.

3 Prather AA, Rabinovitz M, Pollock BG, Lotrich FE: Cytokine-induced depression during IFN- $\alpha$ treatment: the role of IL- 6 and sleep quality. Brain Behav Immun 2009;23:1109-1116.

4 National Center for Complementary and Alternative Medicine: CAM Basics. 2012. Available from: https://nccih.nih.gov/sites/nccam.nih.gov/ files/D347_05-25-2012.pdf.

5 Lee AM, Ng SM, Leung PPY, Chan CLW: Integrative Body-Mind-Spirit Social Work: An Empirically Based Approach to Assessment and Treatment. New York, Oxford University Press, 2009.

6 Chan CL, Ng SM, Ho RT, Chow AY: East meets West: applying Eastern spirituality in clinical practice. J Clin Nurs 2006;15:822-832.

7 Chan $\mathrm{CH}, \mathrm{Ng} \mathrm{EH}$, Chan CL, Ho, Chan TH: Effectiveness of psychosocial group intervention for reducing anxiety in women undergoing in vitro fertilization: a randomized controlled study. Fertil Steril 2006;85:339346.

8 Cheung CK, Christopher B: Validating an American scale in Hong Kong the Center for Epidemiological Studies Depression Scale (CES-D). J Psychol 1998;132:169-186.

9 Tsai PS, Wang SY, Wang MY, Su CT, Yang TT, Huang CJ, Fang SC: Psychometric evaluation of the Chinese version of the Pittsburgh Sleep Quality Index (CPSQI) in primary insomnia and control subjects. Qual Life Res 2005;8:1943-1952.

10 Lund HG, Rybarczyk BD, Perrin PB, Leszczyszyn D, Stepanski E: The discrepancy between subjective and objective measures of sleep in older adults receiving CBT for comorbid insomnia. J Clin Psychol 2013;69: $1108-1120$. 